OPEN ACCESS

Edited by:

Noton Kumar Dutta, Johns Hopkins University,

United States

Reviewed by:

Ananda Shankar Bhattacharjee, Bigelow Laboratory for Ocean

Sciences, United States Junii Xing, Houston Methodist Research Institute, United States

*Correspondence:

$\mathrm{Li} \mathrm{Li}$

annylish@126.com

Zelin Cui

czl_phage@126.com; czl@sjtu.edu.cn

Specialty section: This article was submitted to Antimicrobials, Resistance and Chemotherapy, a section of the journal Frontiers in Microbiology

Received: 11 October 2017 Accepted: 22 June 2018 Published: 23 July 2018

Citation:

Ding B, Li Q, Guo M, Dong K, Zhang Y, Guo X, Liu Q, Li L and Cui Z (2018) Prevention of Dermal Abscess Formation Caused by Staphylococcus aureus Using Phage JD007 in Nude Mice. Front. Microbiol. 9:1553. doi: 10.3389/fmicb.2018.01553

\section{Prevention of Dermal Abscess Formation Caused by Staphylococcus aureus Using Phage JD007 in Nude Mice}

\author{
Bingyu Ding 1,2, Qingtian Li², Mingquan Guo ${ }^{3}, K_{\text {e Dong }}^{3}$, Yan Zhang ${ }^{3}$, Xiaokui Guo ${ }^{3}$, \\ Qingzhong Liu', Li Li ${ }^{1 *}$ and Zelin Cui ${ }^{1 *}$
}

1 Department of Laboratory Medicine, Shanghai General Hospital, Shanghai Jiao Tong University School of Medicine, Shanghai, China, ${ }^{2}$ Department of Laboratory Medicine, Ruijin Hospital, Shanghai Jiao Tong University School of Medicine, Shanghai, China, ${ }^{3}$ Department of Immunology and Microbiology, Shanghai Jiao Tong University School of Medicine, Shanghai, China

Aim: In this study, Staphylococcus phage JD007 bactericidal activity and induced immune responses during treatment were assessed in a dermal abscess model.

Materials and Methods: Dermal abscesses in nude mice were established by injecting a clinical isolate of $S$. aureus SA325 isolated from the back under-dermal abscess of an in-patient.

Results: Phage JD007 was able to inhibit the growth of $S$. aureus SA325 at $\mathrm{MOI}=1$ or 10 , significantly preventing the formation of dermal abscesses. Moderate immune responses were observed in the prevention group through detection of cytokines.

Conclusion: Phage JD007 inhibits the formation of dermal abscesses caused by a clinical S. aureus strain in nude mice without robust immune responses.

Keywords: Staphylococcus aureus, dermal abscess, inhibition, nude mouse, phage JD007

\section{INTRODUCTION}

With the increasing prevalence antibiotic resistant infections, including MRSA (methicillinresistant Staphylococcus aureus) and the emergence of VRSA (vancomycin-resistant S. aureus) (Xiao et al., 2013; Limbago et al., 2014), innovative treatments are urgently needed. Recently, there has been renewed interest in phage therapy (Thiel, 2004; Salmond and Fineran, 2015), which has been proposed as a potential antimicrobial therapy as far back as the 1920's (Salmond and Fineran, 2015). Advances in biotechnology have led to the identification of numerous classes of bacteriophages with different host specificities such as K, GH15, Twort, phiIPLA-RODI, and phiIPLA-C1C (O'Flaherty et al., 2004; Kwan et al., 2005; Gu et al., 2012b; Gutierrez et al., 2015). Furthermore, whole genome sequences of these phages enables us to understand their biology at a much deeper level (Cui et al., 2017b). 
Phages have been used to treat infectious disease in various animal models. A single dose of phage MR10 exhibited efficacy similar to linezolid in resolving the course of hindpaw infection in diabetic animals, suggesting this approach could serve as an effective strategy in treating MRSA mediated foot infections in diabetic individuals (Chhibber et al., 2013). Phage LS2a has been shown to prevent abscess formation in rabbits when injected simultaneously with $S$. aureus into the subcutaneous site (Wills et al., 2005). Phage Kpn5 could potentially be used as stand-alone therapy for the Klebsiella pneumoniae induced burn wound infection, effective even against antibiotic-resistant strains (Kumari et al., 2009). Additionally, in clinical trials, phage products have showed efficacy according to some reports (Marza et al., 2006).

Phage host specificity narrows their use with lysing efficacies that can vary significantly depending on the phage strain. Reports have also documented failures of phage therapy in vivo (Sarker and Brussow, 2016), so, it is necessary to evaluate phages' bacteria killing efficacy both in vitro and in an animal model. Staphylococcus phage JD007, which belongs to family of Myoviridae was identified in 2012 and has been shown to have wide host range (Cui et al., 2012), capable of killing S. aureus of different MLST Types (Cui et al., 2017a). The aim of this study is to evaluate the treatment efficacy of JD007 in dermal abscesses caused by a clinical strain of MRSA and the immune responses elicited during therapy.

\section{MATERIALS AND METHODS}

\section{Animals}

BALB/c nude mice (WT, Shanghai Super B\&K laboratory animal Corp. Ltd., [SCXK(HU)2005-0001], Shanghai, China) were used in this study. Dermal abscesses were stereo tactically induced as described previously (Capparelli et al., 2007). Uninfected mice served as controls. Mice were euthanized on days 1, 3 and 10 after infection. Ethics eligibility of this study was approved by the Shanghai General Hospital Ethics Committee (Shanghai Jiao Tong University School of Medicine). All experiments were performed in accordance with the national animal protection guidelines approved by the local animal ethics committee. Fifty mice were randomly divided into five groups, 10 mice in each group, including control group (no treatment), phage JD007 group (injected with purified phage JD007), infection group (infected by $S$. aureus SA325), prevention group (phage JD007 were injected $1 \mathrm{~h}$ before mice was infected by $S$. aureus SA325), and treatment group (phage JD007 was injected $1 \mathrm{~h}$ after mice were infected by $S$. aureus SA325).

\section{Bacteriophage Purification}

High-titre phage stocks were obtained through amplification in liquid LB (Luria-Bertani) medium supplemented with $10 \mu \mathrm{M}$ $\mathrm{MgCl}_{2}$ and $5 \mu \mathrm{M} \mathrm{CaCl}_{2}$. In culture, S. aureus SA325 was infected phage JD007 at a MOI of 0.1 and incubated at $37^{\circ} \mathrm{C}$ overnight. The visible bacterial lysate in liquid culture were obtained, and then incubated with chloroform (final concentration was $2 \%$ ) for $30 \mathrm{~min}$ with gentle shaking to kill residual bacteria. Remaining bacterial products were removed by centrifugation at 6,500 rpm (Beckman, JA18.0, United States) for $15 \mathrm{~min}$. Phage contained in the supernatant was enriched at $4^{\circ} \mathrm{C}$ overnight using polyethylene glycol (PEG) 8000 (final concentration 10\% $\mathrm{w} / \mathrm{v}$ ), and precipitated at 8,500 rpm for 20 min (Beckman, JA18.0, United States). Afterwards, the pellet was dissolved in TM buffer [(Tris- $\mathrm{Mg}^{2+}$ Buffer) $10 \mathrm{mM}$ Tris- $\mathrm{HCl}$ (pH 7.2-7.5), $100 \mathrm{mM} \mathrm{NaCl}, 10 \mathrm{mM} \mathrm{MgCl}_{2} .5 \mathrm{mM} \mathrm{CaCl}_{2}$ ] and vortexed. PEG 8000 was removed by adding the same volume of chloroform after vortexing. The solution was centrifuged at 4,000 $\mathrm{g} / \mathrm{min}$ for $10 \mathrm{~min}$, and the supernatant containing the phage was
A

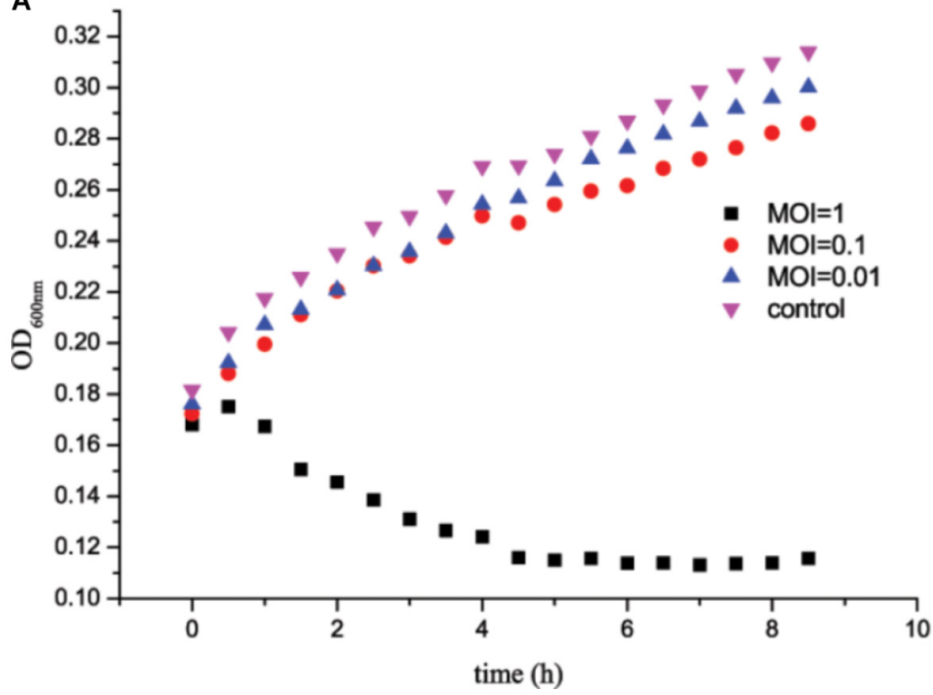

B

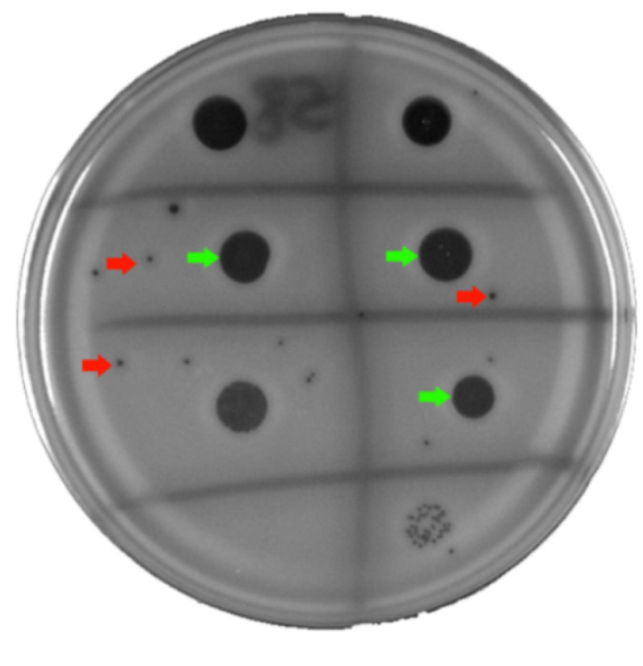

FIGURE 1 | Staphylococcus aureus SA325 growth was inhibited by phage JD007 in vitro. (A) Phage JD007 could inhibit the growth of SA325 at MOI = 1. (B) Inhibition zones and plaques were formed by phage JD007 in two-layer agar plate of SA325 and were marked using green and red arrows, respectively. 

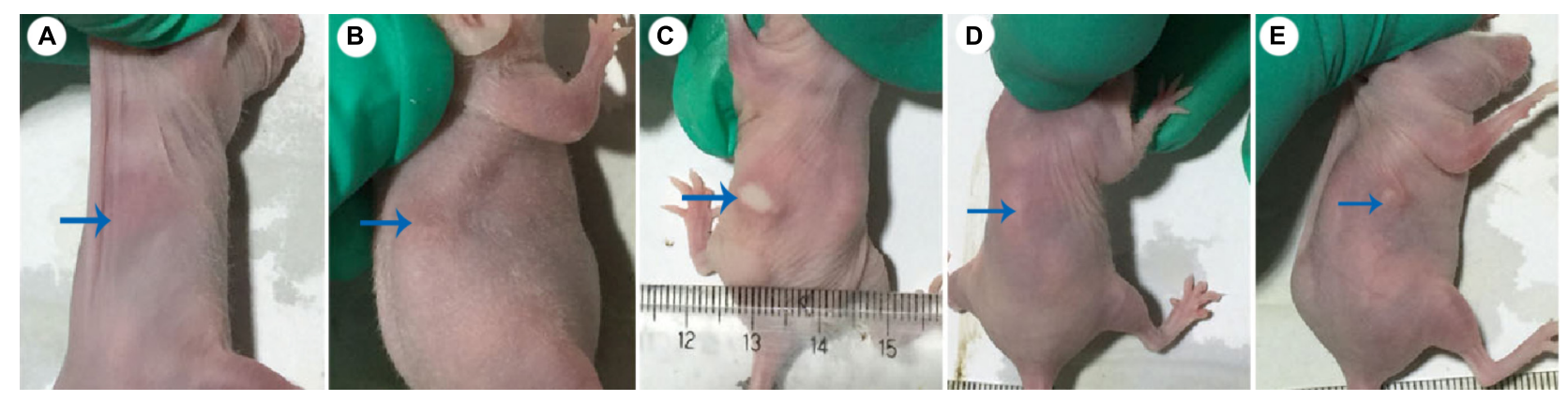

FIGURE 2 | Photos of dermal abscesses in nude BALB/c mice using phage JD007. (A-E) Represented the dermal abscesses of mice individually in control group, phage group, infection group, prevention group, and treatment group. The blue arrows showed the skin sites injected with S. aureus SA325.

isolated. $\mathrm{CsCl}$ was added at a concentration of $0.5 \mathrm{~g}$ per $1 \mathrm{~mL}$, and the phages were purified by discontinuous centrifugation in a $\mathrm{CsCl}$ gradient $\left(1.33,1.45,1.50\right.$, and $\left.1.70 \mathrm{~g} / \mathrm{cm}^{3}\right)$ in $\mathrm{TM}$ buffer in Ultra-Clear tubes (Beckman Coulter, Inc., Fullerton, CA, United States) by centrifuging at $35000 \mathrm{rpm} / \mathrm{min}$ for $4 \mathrm{~h}$. Finally, the layer with enriched phage was obtained with a syringe, dialyzed against TM buffer and stored at $4^{\circ} \mathrm{C}$ (Boulanger, 2009).

\section{S. aureus Strain and Culture Condition}

Staphylococcus aureus strain SA325 (MRSA, confirmed by antibiotic resistant profile) was isolated from an in-patient with a re-occurring back under-dermal abscess due to long-term bed rest. SA325 concentration was calculated by serial dilutions when grown to $\mathrm{OD}_{600 \mathrm{~nm}}=0.4$, and plated uniformly using the glass spreading rod, and cultured overnight at $37^{\circ} \mathrm{C}$, the value of $\mathrm{SA} 325$ per $\mathrm{OD}_{600 \mathrm{~nm}}$ was $9.5 \times 10^{9} \mathrm{CPU} / \mathrm{mL}$.

\section{S. aureus Growth Inhibition Assay}

Drop tests were conducted using two-layer agar plates. S. aureus SA325 was cultured to $\mathrm{OD}_{600 \mathrm{~nm}}=0.4$ in TSB (Tryptic soy broth) supplemented with $10 \mu \mathrm{M} \mathrm{MgCl}$ and $5 \mu \mathrm{M} \mathrm{CaCl}_{2}$. Bacteria grown to logarithmic phase were mixed with $0.7 \%$ agar LB and then poured on the plate of LB agar (1.5\%) uniformly, incubating at room temperature for $30 \mathrm{~min}$. Finally, $3 \mu \mathrm{L}$ of serial dilutions of phage JD007 was dropped on the plate. The inhibition zones were observed after an overnight culture.

The inhibition growth curves for phage JD007 infected S. aureus SA325 were determined using MOIs of $0,0.01,0.1$, and 1 inoculated in 96 well plates separately and incubated at $37^{\circ} \mathrm{C}$. $\mathrm{OD}_{600 \mathrm{~nm}}$ of these cultures was measured every 30 min extending for $8 \mathrm{~h}$. Assay was performed three times $(n=3)$ with three biological replicates.

\section{Mice Dermal Abscess Infection Model}

Staphylococcus aureus was prepared by culturing at $37^{\circ} \mathrm{C}$ in TSB to exponential phase at which time the bacteria was collected and washed twice with PBS with immediate centrifugation. SA325 was re-suspended at final concentration of $1 \times 10^{9} \mathrm{CFUs} / 100 \mu \mathrm{L}$, and kept on ice before injecting nude mice intradermally.
All animal experiments were approved by the Shanghai General Hospital Ethics Committee (Shanghai Jiao Tong University, China), and conducted according to the Chinese Law for Animal Protection. To investigate the antimicrobial efficacy of phage JD007 for subcutaneous abscesses, BALB/c nude mice (6-8 weeks old) were anesthetized by intraperitoneal injection of a saline solution containing fentanyl $(0.05 \mathrm{mg} / \mathrm{kg})$, midazolam $(5 \mathrm{mg} / \mathrm{kg})$, and medetomidine $(0.5 \mathrm{mg} / \mathrm{kg})$. The backs of the animals were disinfected with $70 \%$ ethanol, and $50 \mu \mathrm{L}$ suspension containing $5 \times 10^{8} \mathrm{CFU}$ S. aureus SA325 in PBS were inoculated subcutaneously; $50 \mu \mathrm{L}$ of phage JD007 was injected into the infected region. Mice were weighed before inoculation. Weight and abscess formation were measured daily extending for 10 days. The length (L) and width (W) of abscesses were determined using a caliper. The size of the abscesses was then calculated with the standard formula for area: $\mathrm{V}=[\pi / 2] \times \mathrm{L} \times \mathrm{W}$ (Weinandy et al., 2014).

\section{Immune Response During Treatment Using Staphylococcus Phage JD007}

Three mice in each described above were randomly enrolled and euthanized on the third day of the experimental protocol. Serum from each mouse was obtained, and immune cytokines

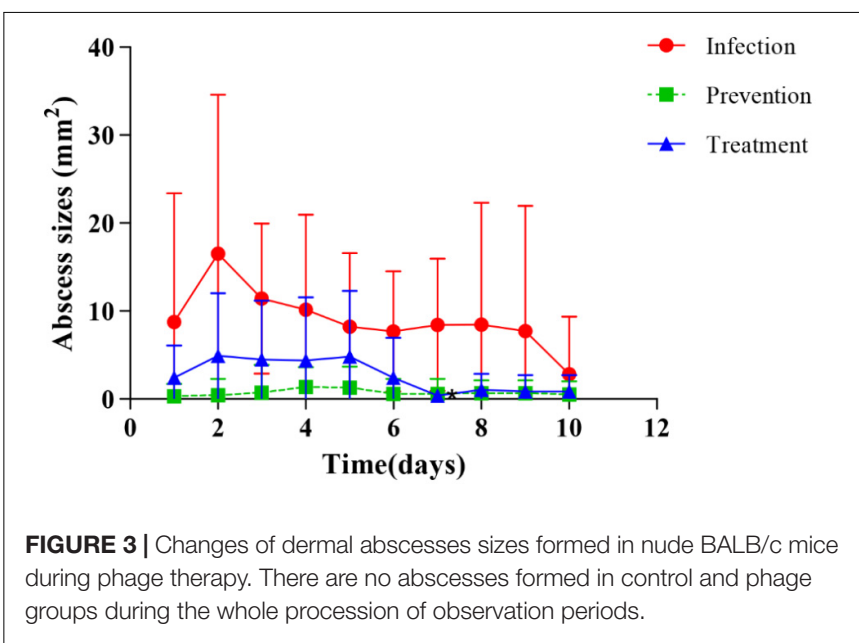




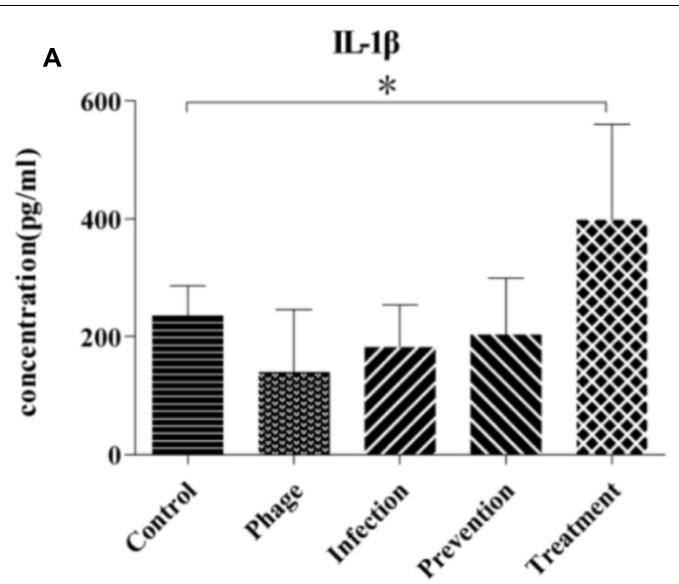

C
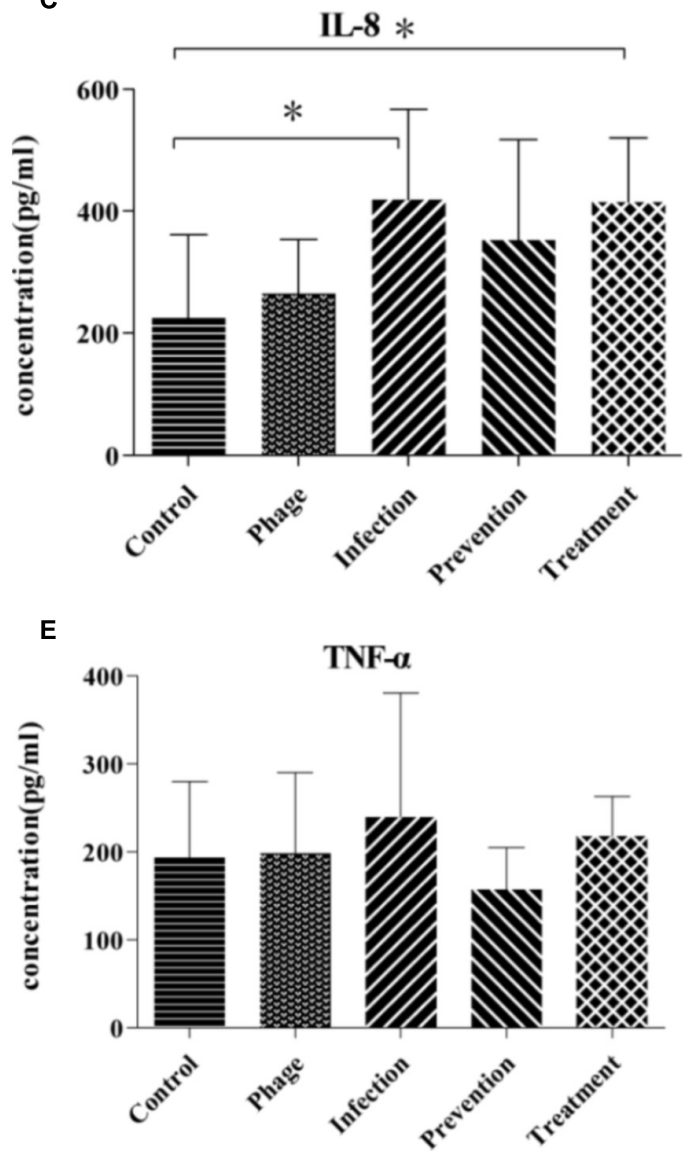

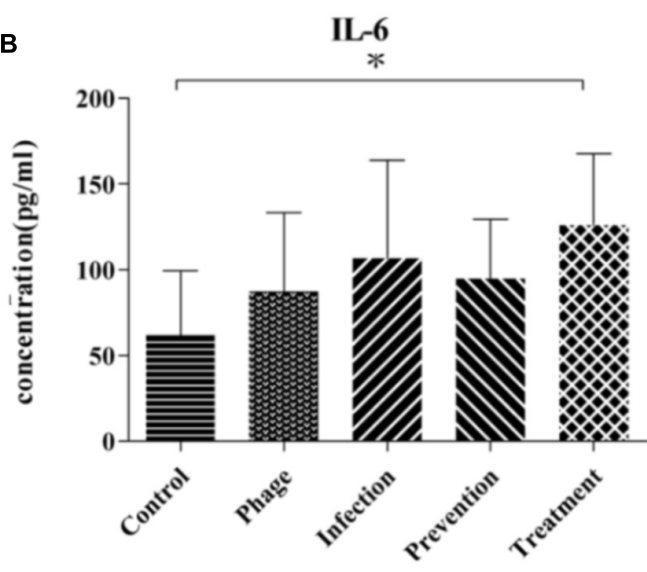

D

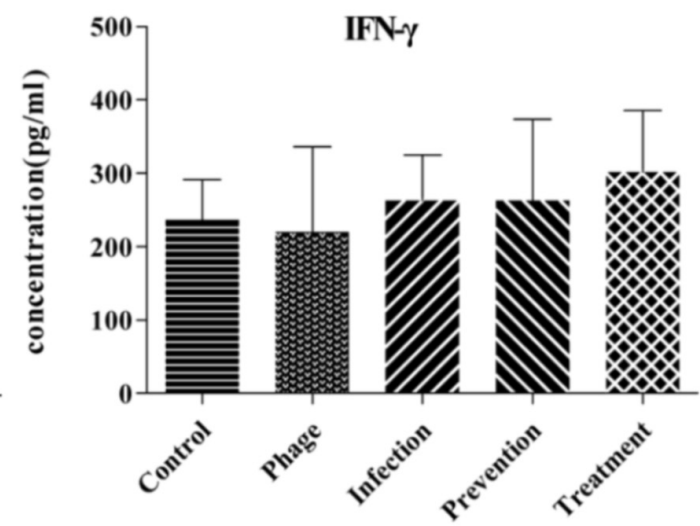

FIGURE 4 | Inflammatory cytokines responses in nude BALB/c mice during phage therapy. (A-E) Are separately represents the sera level of IL-1 $\beta$, IL-6, IL-8, IFN- $\gamma$, and TNF- $\alpha$ of mice from different groups, ${ }^{*} p<0.05$.

(IL-1 $\beta$, IL-6, IL-8, IFN- $\gamma$, and TNF- $\alpha$ ) were measured using ELISA (Beijing 4A Biotech Co., Ltd.).

\section{Statistical Analysis}

A two-tailed unpaired Student's $t$-test was used for statistical analysis with GraphPad Prism Software. P-values of less than 0.05 were considered significant unless specifically indicated otherwise.

\section{RESULTS}

\section{S. aureus SA325 Growth Is Inhibited by}

\section{Phage JD007 in Vitro}

Staphylococcus aureus SA325 was co-cultured with phage JD007 at $37^{\circ} \mathrm{C}$ with co-culture $\mathrm{OD}_{600 \mathrm{~nm}}$ measurements made at $30 \mathrm{~min}$ intervals for $8 \mathrm{~h}$. Results showed that phage JD007 inhibited the 


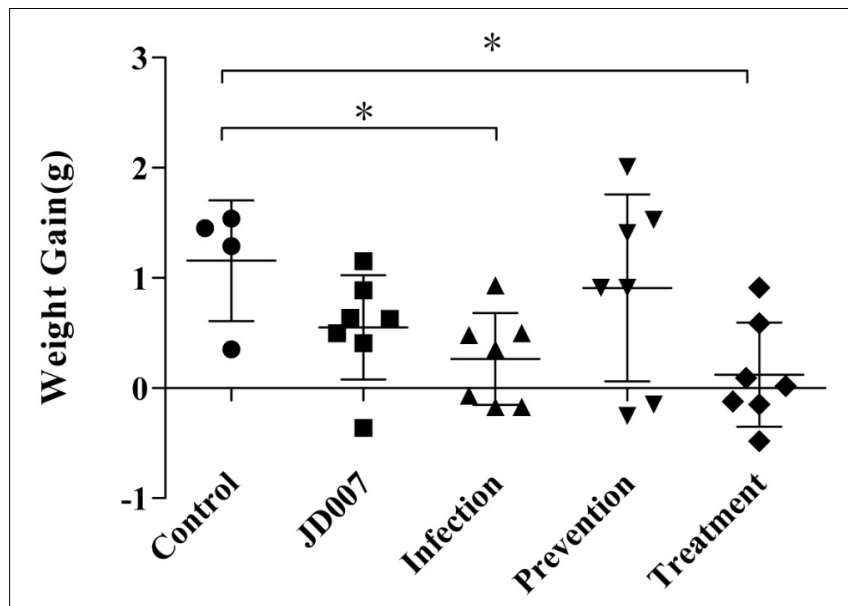

FIGURE 5 | Weights gains in nude BALB/c mice during phage therapy. Body weights gain were calculated by subtracting original body weight from weights on the 10th day. Body weights gains in groups of infection and treatment were significantly lower than that in control group, ${ }^{*} p<0.05$.

growth of $S$. aureus SA325 as early as $1 \mathrm{~h}$ after co-incubation at MOI = 1 (Figure 1). Whereas, S. aureus SA325 grew normally when infected by phage JD007 at lowers MOIs $=0.1$ and 0.01 . The drop test results showed that phage JD007 formed clear inhibition zones in two-layer agar plates.

\section{Phage JD007 Prevents S. aureus SA325-Mediated Dermal Abscess Formation in Nude BALB/c Mice in Vivo}

Dermal abscess formation was established in the back of nude mice and observed daily for 10 days. Results showed that the maximum sizes of dermal abscesses were achieved on the second day following infection. On the seventh day, all of the abscesses were scarred. As shown in Figures 2, 3, we could see no visible dermal abscesses formed in control group, phage group, and prevention group during the entire observation period. On the third day, the abscess sizes tended to decrease in size, and mice's dermal abscess size in the treatment group were significantly smaller than those in the infection group $(p<0.05)$.

\section{Inflammatory Cytokines Responses of Nude BALB/c Mice During Phage Therapy}

Cytokines of IL-1 $\beta$, IL-6, IL-8, IFN- $\gamma$, and TNF- $\alpha$ in the sera were measured using ELISA on the third day. As shown in Figure 4, there were no significant differences between IFN- $\gamma$ and TNF- $\alpha$ among those groups. IL- $1 \beta$, IL- 6 , and IL- 8 in treatment groups were higher than those in control group, whereas there were no significant differences between the negative control and prevention groups.

\section{Weight of Nude BALB/c Mice During Phage Therapy}

Weights of mice in each group were measured daily, encompassing the entire observation period. Body weights gain were calculated by subtracting original body weight from weights on the tenth day. As shown in Figure 5, weight gains in infection group and phage treatment were much lower than that compared with control group. The weight gain in the prevention and phage JD007 groups had no significant difference compared with the PBS control group during treatment.

\section{DISCUSSION}

Staphylococcus aureus SA325 was isolated from a re-occurring back under-dermal abscess of an in-patient with long-term bed rest. In vitro bactericidal activity tests showed that phage JD007 could form a clear inhibition zones using a two-layer soft agar plate assay, and could inhibit growth of SA325 at MOI $=1$ or 10.

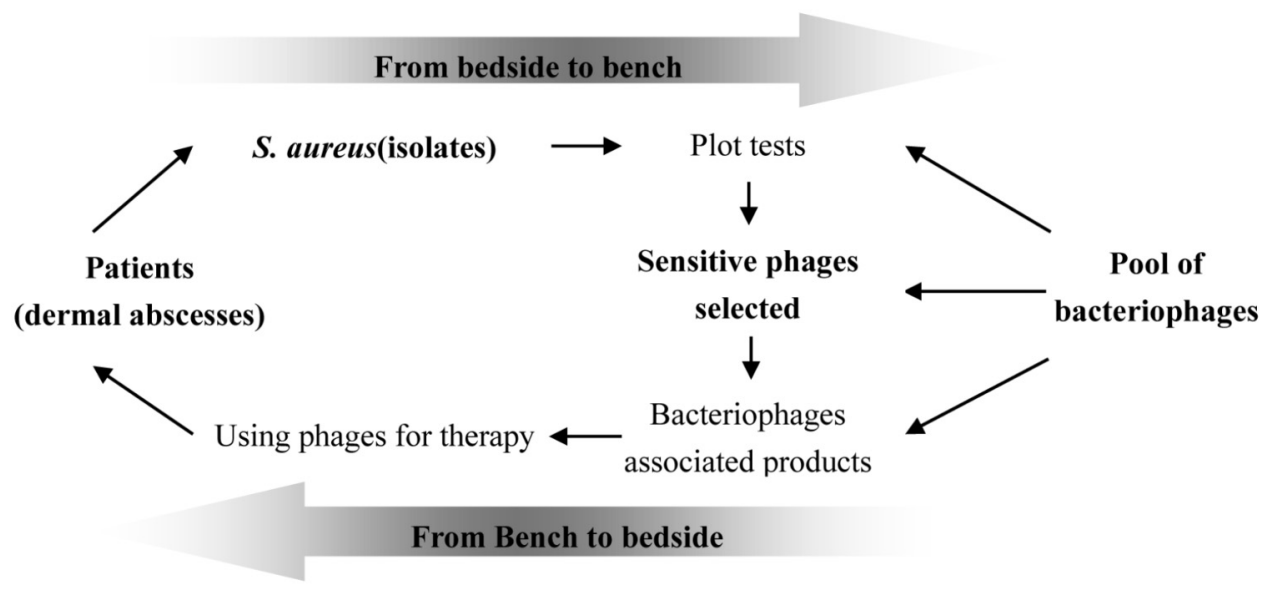

FIGURE 6 | Proposed scheme of selecting phages for therapy. The success of phage therapy needs the pool of phages and their susceptibility to the definite infectious bacterial pathogen at infection sites should be tested previously; the products containing of different phages were produced firstly and the sensitive phages would be chosen for therapy. 
In order to evaluate the ability of phage JD007 to prevent or treat of abscesses caused by $S$. aureus, a dermal abscess model was established in nude mice model using clinical isolate SA325, as reported previously (Malachowa et al., 2013). In this study, we successfully established the dermal abscess infection in nude BALB/c mice using $S$. aureus SA325. Furthermore, the efficacies of treatment of the abscesses using phage JD007 were evaluated. There were no observed abscess formation in the prevention group. The average abscess sizes of phage treatment group were smaller than those of the infection group. The results indicate that phage JD007 prevents formation of abscess. Also, it supports earlier observations on phage $\mathrm{M}^{\text {Sa }}$ inhibition S. aureus dermal abscess formation (Capparelli et al., 2007).

To evaluate the response of mice during prevention and treatment after phage JD007 inoculation, the weight changes were observed during the whole process. As bacteriophages are viruses containing nucleic acid and proteins, they may bring some risk to humans when used for the treatment of bacteremia, as the complex composition of the phage may induce unpredictable immune responses when administered to the blood. The average weights gains in the infection and treatment groups were significantly lower than those in the control, PBS, phage JD007, and prevention groups. Furthermore, immune responses of the mice during treatment were evaluated, mice sera was taken on the third day, and the inflammatory cytokines of IL-1 $\beta$, IL-6, IL-8, IFN- $\gamma$, and TNF- $\alpha$ in serum were measured using ELISA. The cytokines IL- $1 \beta$ and IL- 6 both belong to endogenous pyrogens, and they can be applied to the hypothalamus regulating center to cause fever. IL-1 $\beta$ and IL-6 levels were significantly higher in the treatment group, while there were no significant differences among prevention, infection, or control groups, indicating that phage JD007 treatment may destroy the cells of $S$. aureus robustly, leading to increase of IL- $1 \beta$ and IL- 6 that may cause fever. It's reported that TRIM29 could negatively regulate the proinflammatory cytokine IL- 6 and TNF- $\alpha$ production in response to LPS and bacteria Haemophilus influenzae (Xing et al., 2016). Whether S. aureus infection suppresses the TRIM 29 expression and leads to increased expression of IL- 6 should be further examined. IL-8 was significantly elevated in infection and treatment groups. IL-8 can signal chemotactic neutrophil leukocytes and activate $\mathrm{T}$ cells in the infection sites, clearing the pathogen. IFN- $\gamma$ and TNF- $\alpha$ can activate mononuclear macrophages, which can enhance the phagocytosis and killing functions; TNF- $\alpha$ can directly kill the cell infected by virus. There were no significant changes in any of these groups.

The specificity of bacteriophages is one of important reason hindering clinical use of phage for therapy, though cocktails

\section{REFERENCES}

Boulanger, P. (2009). Purification of bacteriophages and SDS-PAGE analysis of phage structural proteins from ghost particles. Methods Mol. Biol. 502, 227-238. doi: 10.1007/978-1-60327-565-1_13

Capparelli, R., Parlato, M., Borriello, G., Salvatore, P., and Iannelli, D. (2007). Experimental phage therapy against Staphylococcus aureus in mice. Antimicrob. Agents Chemother. 51, 2765-2773. doi: 10.1128/AAC.015 13-06 may overcome this fault ( $\mathrm{Gu}$ et al., 2012a; Mendes et al., 2014). There are numerous public reports describing the success of phage therapy inhibiting infectious diseases caused by dermal associated infections (Vieira et al., 2012; Trigo et al., 2013). We postulate that phage therapy could become an important choice for the treatment of wound infections. As shown in Figure 6, the pool of different phages should be established before using them for therapy; at the same time, the bacteria causing infection should be isolated and its sensitivity to the phages in the pool confirmed. The formula of sensitive phages must be prepared at first; once the bacteria causing the infection is confirmed to be sensitive to phages in the pool, the formula containing the sensitive phage should be chosen for treatment of the infections. The success of phage therapy depends upon the pool of different kinds of bacteriophages.

In summary, phage JD007 could inhibit the growth of S. aureus both in vitro and in vivo. Phage JD007 can prevent abscess formation caused by $S$. aureus isolated from an inpatient's abscess samples through under-dermal injection, and can also significantly reduce the severity of dermal abscess caused by $S$. aureus. In conclusion, phage JD007 is a promising candidate phage for use in preventing dermal abscess.

\section{AUTHOR CONTRIBUTIONS}

ZC, QTL, and BD carried out the experiments and drafted the manuscript. QTL, KD, BD, QZL, YZ, and MG participated in the mice model experiments. ZC, QTL, XG, and LL participated in the design of the study and performed the statistical analysis. ZC, QTL, and LL conceived the study, participated in its design, and coordinated to help draft the manuscript.

\section{FUNDING}

This work was sponsored in part by the National Natural Science Foundation of China (No. 31500154), the fund of Shanghai Health and Family Planning Committee (No. 201440289), and the outstanding medical youth program A of Shanghai General Hospital (No. 06N1702002).

\section{ACKNOWLEDGMENTS}

Thanks Luis J. Cocka for suggestions of modification manuscript.

Chhibber, S., Kaur, T., and Sandeep, K. (2013). Co-therapy using lytic bacteriophage and linezolid: effective treatment in eliminating methicillin resistant Staphylococcus aureus (MRSA) from diabetic foot infections. PLoS One 8:e56022. doi: 10.1371/journal.pone.0056022

Cui, Z., Feng, T., Gu, F., Li, Q., Dong, K., Zhang, Y., et al. (2017a). Characterization and complete genome of the virulent Myoviridae phage JD007 active against a variety of Staphylococcus aureus isolates from different hospitals in Shanghai, China. Virol. J. 14:26. doi: 10.1186/s12985-0170701-0 
Cui, Z., Guo, X., Dong, K., Zhang, Y., Li, Q., Zhu, Y., et al. (2017b). Safety assessment of Staphylococcus phages of the family Myoviridae based on complete genome sequences. Sci. Rep. 7:41259. doi: 10.1038/srep41259

Cui, Z., Song, Z., Wang, Y., Zeng, L., Shen, W., Wang, Z., et al. (2012). Complete genome sequence of wide-host-range Staphylococcus aureus phage JD007. J. Virol. 86, 13880-13881. doi: 10.1128/JVI.02728-12

Gu, J., Liu, X., Li, Y., Han, W., Lei, L., Yang, Y., et al. (2012a). A method for generation phage cocktail with great therapeutic potential. PLoS One 7:e31698. doi: 10.1371/journal.pone.0031698

Gu, J., Liu, X., Lu, R., Li, Y., Song, J., Lei, L., et al. (2012b). Complete genome sequence of Staphylococcus aureus bacteriophage GH15. J. Virol. 86, 8914-8915. doi: 10.1128/JVI.01313-12

Gutierrez, D., Vandenheuvel, D., Martinez, B., Rodriguez, A., Lavigne, R., and Garcia, P. (2015). Two phages, phiIPLA-RODI and phiIPLA-C1C, lyse monoand dual-staphylococcal biofilms. Appl. Environ. Microbiol. 81, 3336-3348. doi: 10.1128/AEM.03560-14

Kumari, S., Harjai, K., and Chhibber, S. (2009). Efficacy of bacteriophage treatment in murine burn wound infection induced by Klebsiella pneumoniae. J. Microbiol. Biotechnol. 19, 622-628.

Kwan, T., Liu, J., DuBow, M., Gros, P., and Pelletier, J. (2005). The complete genomes and proteomes of 27 Staphylococcus aureus bacteriophages. Proc. Natl. Acad. Sci. U.S.A. 102, 5174-5179. doi: 10.1073/pnas.0501140102

Limbago, B. M., Kallen, A. J., Zhu, W., Eggers, P., McDougal, L. K., and Albrecht, V. S. (2014). Report of the 13th vancomycin-resistant Staphylococcus aureus isolate from the United States. J. Clin. Microbiol. 52, 998-1002. doi: 10.1128/JCM.02187-13

Malachowa, N., Kobayashi, S. D., Braughton, K. R., and DeLeo, F. R. (2013). Mouse model of Staphylococcus aureus skin infection. Methods Mol. Biol. 1031, 109-116. doi: 10.1007/978-1-62703-481-4_14

Marza, J. A., Soothill, J. S., Boydell, P., and Collyns, T. A. (2006). Multiplication of therapeutically administered bacteriophages in Pseudomonas aeruginosa infected patients. Burns 32, 644-646. doi: 10.1016/j.burns.2006.02.012

Mendes, J. J., Leandro, C., Mottola, C., Barbosa, R., Silva, F. A., Oliveira, M., et al. (2014). In vitro design of a novel lytic bacteriophage cocktail with therapeutic potential against organisms causing diabetic foot infections. J. Med. Microbiol. 63, 1055-1065. doi: 10.1099/jmm.0.071753-0

O'Flaherty, S., Coffey, A., Edwards, R., Meaney, W., Fitzgerald, G. F., and Ross, R. P. (2004). Genome of staphylococcal phage K: a new lineage of Myoviridae infecting gram-positive bacteria with a low $\mathrm{G}+\mathrm{C}$ content. J. Bacteriol. 186, 2862-2871. doi: 10.1128/JB.186.9.2862-2871.2004

Salmond, G. P., and Fineran, P. C. (2015). A century of the phage: past, present and future. Nat. Rev. Microbiol. 13, 777-786. doi: 10.1038/nrmicro3564
Sarker, S. A., and Brussow, H. (2016). From bench to bed and back again: phage therapy of childhood Escherichia coli diarrhea. Ann. N. Y. Acad. Sci. 1372, 42-52. doi: 10.1111/nyas. 13087

Thiel, K. (2004). Old dogma, new tricks-21st Century phage therapy. Nat. Biotechnol. 22, 31-36. doi: 10.1038/nbt0104-31

Trigo, G., Martins, T. G., Fraga, A. G., Longatto-Filho, A., Castro, A. G., Azeredo, J., et al. (2013). Phage therapy is effective against infection by Mycobacterium ulcerans in a murine footpad model. PLoS Negl. Trop. Dis. 7:e2183. doi: 10.1371/ journal.pntd.0002183

Vieira, A., Silva, Y. J., Cunha, A., Gomes, N. C., Ackermann, H. W., and Almeida, A. (2012). Phage therapy to control multidrug-resistant Pseudomonas aeruginosa skin infections: in vitro and ex vivo experiments. Eur. J. Clin. Microbiol. Infect. Dis. 31, 3241-3249. doi: 10.1007/s10096-0121691-x

Weinandy, F., Lorenz-Baath, K., Korotkov, V. S., Bottcher, T., Sethi, S., Chakraborty, T., et al. (2014). A beta-lactone-based antivirulence drug ameliorates Staphylococcus aureus skin infections in mice. ChemMedChem 9, 710-713. doi: 10.1002/cmdc.201300325

Wills, Q. F., Kerrigan, C., and Soothill, J. S. (2005). Experimental bacteriophage protection against Staphylococcus aureus abscesses in a rabbit model. Antimicrob. Agents Chemother. 49, 1220-1221. doi: 10.1128/AAC.49.3.12201221.2005

Xiao, M., Wang, H., Zhao, Y., Mao, L. L., Brown, M., Yu, Y. S., et al. (2013). National surveillance of methicillin-resistant Staphylococcus aureus in China highlights a still-evolving epidemiology with 15 novel emerging multilocus sequence types. J. Clin. Microbiol. 51, 3638-3644. doi: 10.1128/JCM.013 75-13

Xing, J., Weng, L., Yuan, B., Wang, Z., Jia, L., Jin, R., et al. (2016). Identification of a role for TRIM29 in the control of innate immunity in the respiratory tract. Nat. Immunol. 17, 1373-1380. doi: 10.1038/ni.3580

Conflict of Interest Statement: The authors declare that the research was conducted in the absence of any commercial or financial relationships that could be construed as a potential conflict of interest.

Copyright (C) 2018 Ding, Li, Guo, Dong, Zhang, Guo, Liu, Li and Cui. This is an open-access article distributed under the terms of the Creative Commons Attribution License (CC BY). The use, distribution or reproduction in other forums is permitted, provided the original author(s) and the copyright owner(s) are credited and that the original publication in this journal is cited, in accordance with accepted academic practice. No use, distribution or reproduction is permitted which does not comply with these terms. 